\title{
Quantum dynamics of few dipolar bosons in a double-well potential
}

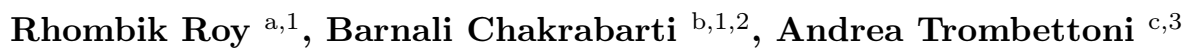 \\ ${ }^{1}$ Department of Physics, Presidency University, 86/1 College Street, Kolkata 700073, India \\ ${ }^{2}$ The Abdus Salam International Center for Theoretical Physics, I34100 Trieste, Italy \\ ${ }^{3}$ CNR-IOM DEMOCRITOS Simulation Centre and SISSA, Via Bonomea 265, I-34136 Trieste, Italy
}

July 28, 2021

\begin{abstract}
We study the few-body dynamics of dipolar bosons in one-dimensional double-wells. Increasing the interaction strength, by investigating one-body observables we study in the considered few-body systems tunneling oscillations, self-trapping and the regime exhibting an equilibrating behaviour. The corresponding two-body correlation dynamics exhibits a strong interplay between the interatomic correlation due to non-local nature of the repulsion and the inter-well coherence. We also study the link between the correlation dynamics and the occupation of natural orbitals of the one-body density matrix.
\end{abstract}

\section{Introduction}

The study of the tunneling quantum dynamics of ultracold atoms in double-well potentials is a major tool to investigate macroscopic coherent effects and manybody properties [1]. Given the very high level of manipulation and control of quantum gases, the direct observations of tunneling and nonlinear self-trapping is experimentally possible for ultracold bosons $[2,3,4,5,6,7$, 8, 9, 10,11] and fermions [12,13,14,15] both for doubleand multi-well potentials.

When in the uncoupled wells the system can be described by macroscopic wavefunctions, one has that the superfluid dynamics in the double-well potential realizes the so-called Bosonic Josepshon junction (BJJ). For a Bose-Einstein condensate in a double-well potential, interactions between the particles plays a crucial role. When the initial population imbalance of the two wells is below a critical value, Josephson oscillations are predicted [1,16]. These two different dynamical regimes, Josephson oscillations and nonlinear self-trapping, for a BEC in a double-well potential are commonly described at the mean-field level 1, 17, 18, 19,20. To study effects of quantum fluctuations and collapse-revival, one may

${ }^{\mathrm{a}} \mathrm{e}$-mail: rhombik.rs@presiuniv.ac.in

be-mail: barnali.physics@presiuniv.ac.in

${ }^{\mathrm{c}} \mathrm{e}$-mail: andreatr@sissa.it resort to quantum two-mode models $[21,22,23,24,25$. 26, 27, 28]. Beyond mean-field effects are very important for coupled one-dimensional systems [29, 30, 31, 32, 33, 34.

The solution of the time-dependent many-boson Schrödinger equation sheds light to several intriguing features, including the role played by natural orbitals (i.e., the eigenstates of one-body density matrix) corresponding to the eigenvalues smaller than the largest one [35, 36, 37, 38. The many-body tunneling dynamics in one dimensional double-well trap with contact interactions has been also studied considering the full crossover from weak interaction to the fermionization limit, and also attractive interactions with [39,40,41, 42,43].

The experimental realization of dipolar gases, achieved with ultracold dipolar atoms of chromium [44,45], dysprosium [46] and erbium atom [47], further enlarged the range of phenomena that can be studied [48,49], such as coupled one-dimensional systems with tunable dipolar interaction [50]. The non-local nature of the dipolar interaction motivated several studies having as a goal the comparison of results for the quantum dynamics with non-local interactions with the corresponding short-range findings and with recent results for quantum systems with long-range couplings $[51,52,53,54,55$, $56,57,58,59,60$. 
The ground state properties of ultracold trapped bosons with dipolar interactions have been studied extensively both at mean-field level and in the one-dimensional limit $[61,62,63,64,65,66,67,68,69,70$ (see more refs. in [48, 49]). Properties of dipolar bosons in a double well potential were studied by mean-field [71] and by the quntum two-mode model 72. The many-body dynamics of bosons with non-local interaction has been recently studied in [73,74, addressing the effect of a finite-range interaction on density oscillation, collapse and self-trapping and focusing on the comparison between the manybody and mean-field properties. The tunneling dynamics is studied for an interaction potential modeled as $W(r)=\frac{\lambda_{0}}{\sqrt{(r / D)^{2 n}+1}}$ with tunable strength $\lambda_{0}$ and half width $D$.

In the present article we would like to address the quantum dynamics of few dipolar bosons in a onedimensional double-well. The few body systems we are going to study have the advantage that it is possible to investigate in detail the full one-body density matrix and correlation functions. In this way the effect of the non-local nature of the interaction and of the one-dimensional nature of the potential on the quantum dynamics can be investigated in details, and the dynamical regimes determined by them can be visualized and understood. This will give insights on the more complicated problem of many-body dynamics of one-dimensional dipolar atoms in double-well dynamics and their quantum tunneling effects to characterize what are the differences and anologes with the usual mean-field findings. Moreover, our results will be lead us to to make a qualitative link between the observed dynamics of quantum correlations with the interatomic and inter-well correlations. The observed tunneling dynamics is also connected with the occupation of the natural orbitals.

The non-local, dipolar interaction is modeled as

$$
W(r)=\frac{g_{d}}{r^{3}+\alpha_{0}}
$$

( $r=\left|x_{i}-x_{j}\right|$ being the distance between two particles located in $x_{i}$ and $x_{j}$ ), where $g_{d}$ is the strength of dipolar interaction and $\alpha_{0}$ is a cutoff parameter to avoid the divergence at $x_{i}=x_{j}$ (see the corresponding discussion in 62]). $g_{d}$ is determined by the scattering length and transverse confinement [75]. In the follwing we keep $g_{d}>0$, corresponds to repulsive dipolar bosons. We prepare the initial state with complete population imbalance in the asymmetric double-well, with all bosons stayng in the right well. The asymmetric double-well is modeled for simplicity as a superposition of three terms: a harmonic oscillator, a Gaussian with a central barrier and a linear external potential 39. In dimensionless units $V$ reads

$$
V(x)=\frac{1}{2} x^{2}+V_{0} \frac{e^{-\frac{x^{2}}{\sigma^{2}}}}{\sqrt{\pi} \sigma}-d x,
$$

with the parameter $d$ is initally kept sufficiently large to make the right well energetically favorable. The initial state is prepared with practically all atoms in the right well. To study the dynamics in the symmetric doublewell, $d$ is then instantaneously ramped down to zero at $t=0$. We solve the time-dependent Schrödinger equation for $N=6$ dipolar bosons. The tunneling dynamics is monitored by the one-body density, the population in the right well, the population imbalance and the twobody density for various choices of $g_{d}$. At $g_{d}=0$, we observe pure Rabi oscillation [76]. With very weak interactions, the Rabi oscillation is modified in amplitude. With larger $g_{d}$, self-trapping is observed. When $g_{d}$ is sufficiently strong, a new regime sets in and the bosons appear to equilibrate in two wells. These four kind of dynamics are further linked with intricated interplay interatomic correlation and inter-well coherence. We observe that, for the considered number of particles, the many-body state occupies many natural orbitals in this one-dimensional setup also when $g_{d}$ is small. A marked occupation of different natural orbitals is observed for very high value of $g_{d}$, where a vanishig population imbalance is observed for large times.

The structure of the paper is as follows. In Sec. 2 we discuss the setup and give a brief introduction to the used numerical method. In Sec. 3 we present our results, while summary and conclusions are given in Sec. 4.

\section{The model}

The time-dependent many-body Schrödinger equation for $N$ interacting bosons is given by

$$
i \partial_{t}|\Psi\rangle=\hat{H}|\Psi\rangle
$$

(with $\hbar=1$ ). Here, the Hamiltonian $\hat{H}$ is given by

$$
\hat{H}\left(x_{1}, x_{2}, \ldots, x_{N}\right)=\sum_{i=1}^{N} \hat{h}\left(x_{i}\right)+\sum_{i<j=1} \hat{W}\left(x_{i}-x_{j}\right) .
$$

$\hat{h}(x)=\hat{T}(x)+\hat{V}(x)$ is the one-body term in the Hamiltonian, with the potential $V$ given by Eq. (2) with $d=0$ during the dynamics and chosen (for $t<0$ ) to be large to determine the initial state as described in the Introduction. $W$ is the interparticle potential, given in Eq. (1). The total Hamiltonian $\hat{H}$ is written in dimensionless units, as obtained by dividing the dimensionful 
Hamiltonian by $\frac{\hbar^{2}}{m L^{2}}$ ( $m$ is the mass of the bosons and $L$ is an appropriately chosen length scale).

In the following Eq. (4) is solved by the numerical many-body method called multi-configuration timedependent Hartree method for bosons (MCTDHB) 77. [78] implemented in the MCTDH-X software [79, 80, 82 , 81. The MCTDHB has been extensively used in different trapping potentials and interactions $83,84,85$, 86, 87, 88, 89, 90, 91, 92, 93, 94, 95, 96, 97. MCTDH-X has been verified against experimental predictions [98] and is reviewed in 99 .

The many-body wavefunction in a complete set of time-dependent permanents, distributing $N$ bosons in $M$ time-dependent single particle orbitals. Thus the ansatz for the many-body wavefunction is

$$
|\Psi(t)\rangle=\sum_{\bar{n}} C_{\bar{n}}(t)|\bar{n} ; t\rangle
$$

with

$$
|\bar{n} ; t\rangle=\prod_{i=1}^{M}\left(\frac{\left(b_{i}^{\dagger}(t)\right)^{n_{i}}}{\sqrt{n_{i} !}}\right)|v a c\rangle .
$$

In Eq. (5) the summation runs over all possible configurations

$$
N_{\text {conf }}=\left(\begin{array}{c}
N+M-1 \\
N
\end{array}\right)
$$

The $|\bar{n} ; t\rangle=\left|n_{1}, \ldots, n_{M} ; t\right\rangle$ (with $\sum_{i} n_{i} \equiv N$ ) are the time-dependent permanents, the operators $b_{i}^{\dagger}(t)$ create a boson in the $i$ th single particle state $\phi_{i}(x, t)$, and $|v a c\rangle$ is the vacuum. The bosonic annihilation and corresponding creation operators obey the canonical commutation relation $\left[b_{k}, b_{j}^{\dagger}(t)\right]=\delta_{k j}$ at any time. It is important to emphasize that in the ansatz (5) both the expansion coefficients $\left\{C_{\vec{n}}(t) ; \sum_{i} n_{i}=N\right\}$ and the orbitals $\left\{\phi_{i}\left(x_{i}, t\right)\right\}_{i=1}^{M}$ that build up the permanents $|\bar{n} ; t\rangle$ are time-dependent and fully variationally optimized quantities.

In the limit of $M \rightarrow \infty$, the expansion (5) is exact. However, we limit the size of the Hilbert space during our computation requiring proper convergence. As permanents are time-dependent, a given degree of accuracy is reached with a shorter expansion compared to time-independent basis. To solve the time-dependent wavefunction $\Psi(t)$, we utilize the time-dependent variational principle [100,101]. We substitute the many-body ansatz into the functional action of the time-dependent Schrödinger equation and require the stationarity of the functional action with respect to $\left\{C_{\bar{n}}(t)\right\}$ and $\left\{\phi_{n}(\bar{r}, t)\right\}$ which lead to the working equations of the MCTDHB method 78, 81.

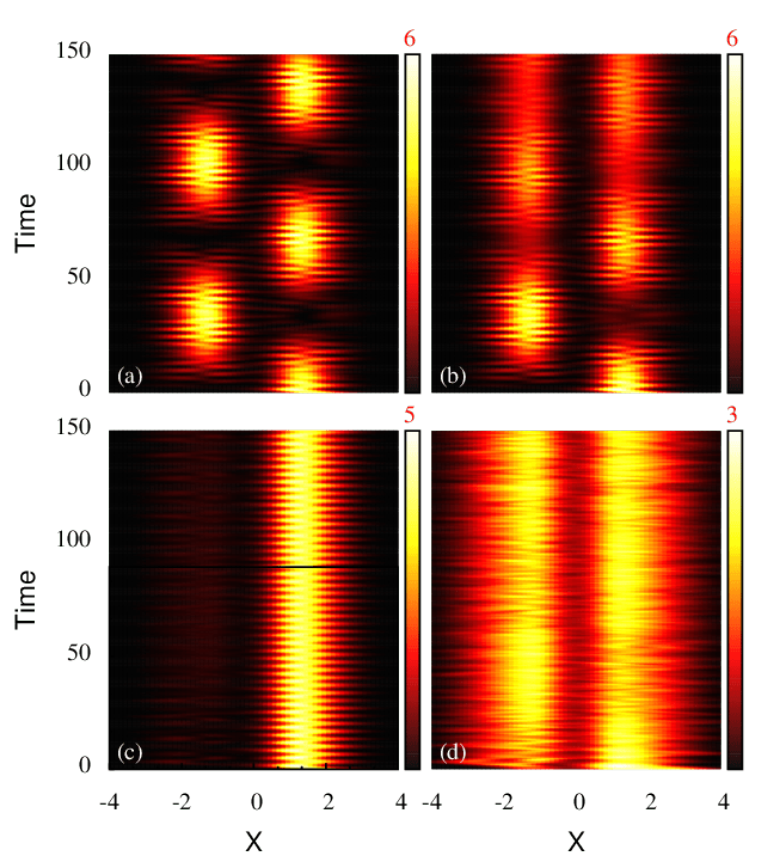

Fig. 1 Tunneling dynamics of $N=6$ interacting dipolar bosons in the double-well potential for different interaction strengths $g_{d}$. The population of the left and right well as a function of time is presented. (a) $g_{d}=0$, Rabi oscillations. (b) $g_{g}=0.002$, deformed Rabi oscillations. (c) $g_{d}=0.02$, self-trapping in right well. (d) $g_{d}=0.2$, equilibration in both wells.

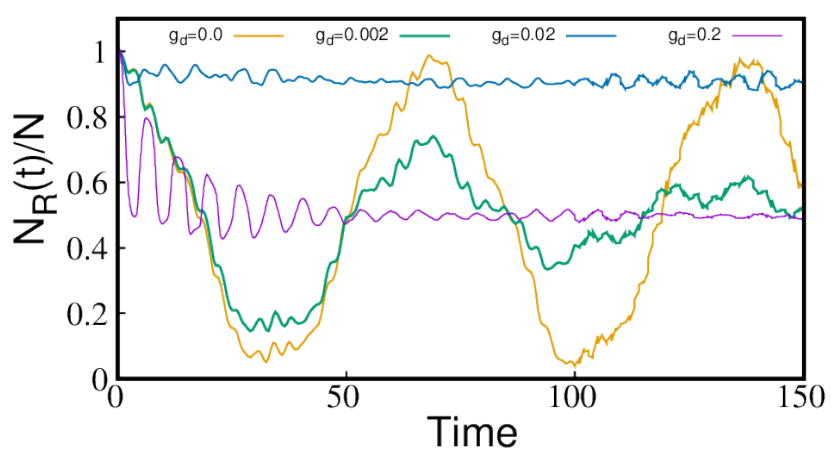

Fig. 2 Time evolution of the relative population in the righthand well $\left(\frac{N_{R}(t)}{N}\right)$ for $N=6$ dipolar bosons in double-well potential for different interaction strengths $g_{d}$.

\section{Results}

To prepare the initial state, we propagate the equations of motion in imaginary time starting for an initial guess, so to have the ground state of $N=6$ interacting bosons in the right well of the asymmetric double-well $V(x)$ given by Eq. (2) at $t<0$. Chosen values of the 
parameters are $V_{0}=8.0$ and $\sigma=0.7$. To study the dynamics in the symmetric double-well we ramp down $d \rightarrow 0$ at $t=0$. We consider increasing values of $g_{d}$. We choose $g_{d}=0,0.002,0.02,0.2$ in our numerical simulations, as represenatives of the different regimes we observed. Throughout our work, we perform the computation with $M=10$ orbitals to have converged results. We observed negligible quantitative difference between the computed quantities with $M=10$ and $M=12$ orbitals. Convergence is also assured as the occupation in the last orbital is close to zero.

We observe that for the considered values $g_{d}=$ $0,0.002,0.02,0.2$, the ratio between the height of the potential, $V_{0}$, and the chemical potential, $\mu$ is, respectively, 3.61, 3.44, 2.52, 0.69. Since in general the twomode ansatz is expected to reasonably work when $V_{0} / \mu \gtrsim$ 1 , then varying $g_{d}$ we pass from a regime in which $V_{0} / \mu$ is well larger than 1 to the one in which smaller than 1 .

The tunneling dynamics is presented through the study of the following quantities:

(a) One-body tunneling dynamics:

From the many-body wavefunction $\psi\left(x_{1}, x_{2}, \ldots, x_{N} ; t\right)$, the reduced one-body density matrix $\rho^{(1)}$ is calculated as

$$
\begin{array}{r}
\rho^{(1)}\left(x^{\prime} \mid x ; t\right)=N \int d x_{2} d x_{3} \ldots d x_{N} \\
\psi^{*}\left(x^{\prime}, x_{2}, \ldots, x_{N} ; t\right) \psi\left(x, x_{2}, \ldots, x_{N} ; t\right) .
\end{array}
$$

Its diagonal gives the one-body density $\rho(x, t)$, defined as

$$
\begin{array}{r}
\rho(x ; t)=N \int d x_{2} d x_{3} \ldots d x_{N} \psi^{*}\left(x, x_{2}, \ldots, x_{N} ; t\right) \\
\psi\left(x, x_{2}, \ldots, x_{N} ; t\right) .
\end{array}
$$

It gives the density at the position $x$ and time $t$, when the contribution of other particles is traced out.

For each choice of $g_{d}$, we calculate $\rho(x, t)$ till time $t=150$. We present $\rho(x, t)$ in Fig 1 for different values of $g_{d}$. Comparison is made with the non-interacting case. In absence of any interactions $\left(g_{d}=0\right)$, the atoms simply exhibit Rabi oscillation between both wells. For $g_{d}=0.002$ (a very small value), we observe tunneling dynamics, with the frequency and amplitude of oscillations significantly affected by the repulsive tail of nonlocal interaction. We do the computation for several other number of dipolar bosons, $N=3,4,5$ and observe the same dynamics. For $g_{d}=0.02$, the tunneling dynamics exhibits a kind of self-tapping. The population in the right well is nearly stationary even for long time. The self-trapping at a relatively weak interaction appears to be a consequence of long-range repulsive tail of the dipole interaction. For contact interaction (not shown here), we find the self-trapping behaviour but for much higher interaction strength. We report also the computation with $N=3,4,5$. We observe clear signature of self-trapping at short time dynamics. However, as expected, due to quantum fluctuations, the bosons do not remain in the self-trapped state for very long time irrespective of the particle number. For much higher interaction strength, with $g_{d}=0.2$, we observe equilibration dynamics. An equal number of particles settle in the two wells.

\section{(b) Population imbalance:}

We further calculate the population in the right well using

$$
N_{R}(t)=\int_{0}^{\infty} \rho(x, t) d x,
$$

and plot $\frac{N_{R}(t)}{N}$ in Fig. 2 for the same choice of the values of $g_{d}$. For $g_{d}=0$, we again observe pure Rabi oscillations as expected. For $g_{d}=0.002$, as we introduce small correlations, we observe two different scale of dynamics. Up to a time $t \simeq 80$, the number of bosons in the right well fluctuates. At longer time dynamics, we observe a trend of partial equilibration, with the value of $\frac{N_{R}(t)}{N}$ trying to saturate at 0.5 . When $g_{d}=0.02$, which is ten times larger than the previous one, we observe a single scale of dynamics, with $\frac{N_{R}(t)}{N}$ remaining close to unity throughout the dynamics. From the direct calculation of the population in the right well, we estimate that for this choice of $g_{d}$, the self-trapping is close to $90 \%$. When we analyze the dynamics with $g_{d}=0.2$, the system shows a different type of single scale dynamics i.e. equilibration. With $N_{L}(t)=\int_{-\infty}^{0} \rho(x, t) d x$, we find in this state $N_{L} \simeq N_{R} \simeq 3$. Thus when the strength of dipolar interaction is tuned, we observe four kinds of dynamical features: pure Rabi oscillation, deformed Rabi oscillation with some signature of partial equilibration at large times, nonlinear self-trapping and equilibration.

We also compute the population imbalance as

$$
Z(t)=\frac{N_{R}(t)-N_{L}(t)}{N}
$$

and its average value calculated as

$$
Z_{\text {avg }}=\frac{1}{t} \int_{0}^{t} Z\left(t^{\prime}\right) d t^{\prime}
$$

We plot $Z_{a v g}$ as a function of $g_{d}$ in Fig. 3. It exhibits a kind of crossover between the four kind of dynamics reported above. $Z_{a v g}$ is zero for the two extreme cases, namely Rabi oscillation for non-interacting limit and equilibration dynamics for strong interaction. Whereas 


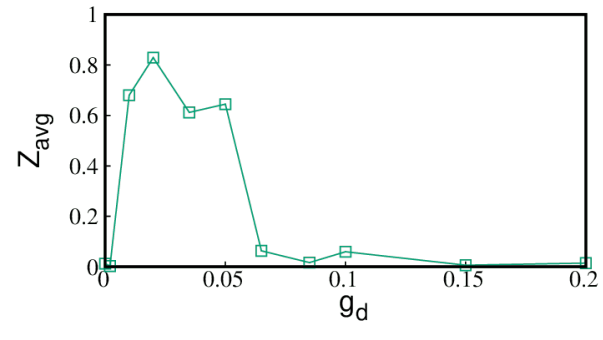

Fig. 3 Time evolution of average value of population imbalance as a function of the interaction strength $g_{d}$. The peak point corresponds to the maximum self-trapping.

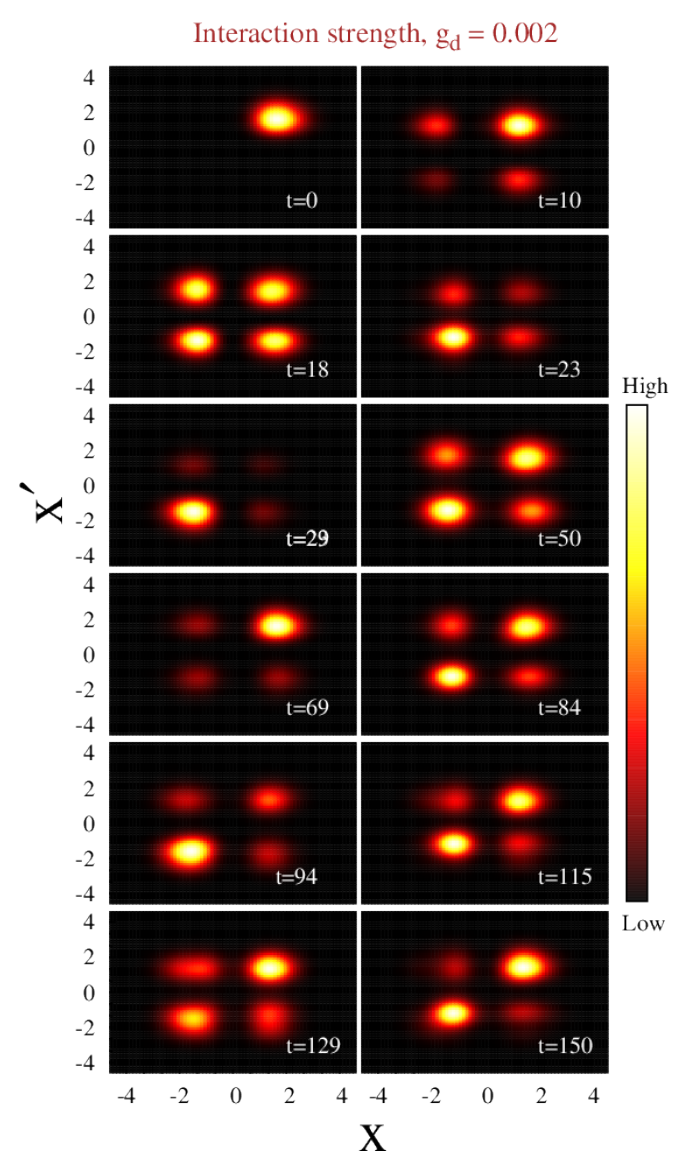

Fig. 4 Time evolution of the two-body density for $g_{d}=$ 0.002 .

maximum population imbalance occurs at some interaction strength $g_{d}^{c}$ which corresponds to self-trapping. Thus the width of the curve qualitatively gives an estimate to have the interaction regime for self-trapping.

\section{(c) Two-body correlation dynamics:}

Next we calculate the time evolution of the two-body density and investigate how the effect of two-body correlation comes in the dynamics increasing $g_{d}$. The sec-

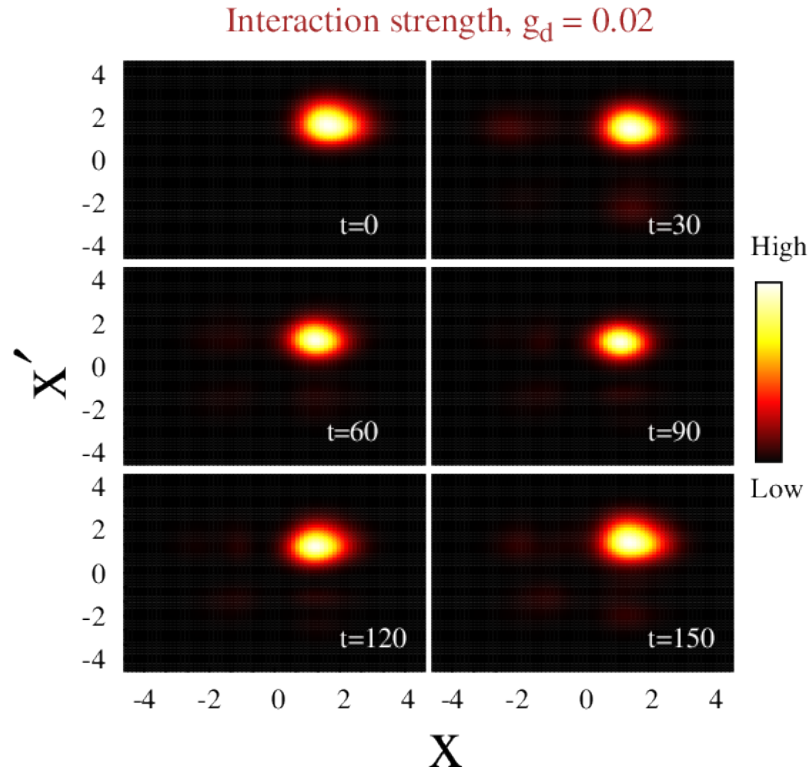

Fig. 5 Time evolution of spatial two-body density for $g_{d}=$ 0.02 .

ond order reduced density matrix is defined as

$$
\begin{array}{r}
\rho^{(2)}\left(x_{1}^{\prime}, x_{2}^{\prime} \mid x_{1}, x_{2} ; t\right)=N(N-1) \int d x_{3} d x_{4} \ldots . d x_{N} \\
\psi^{*}\left(x_{1}^{\prime}, x_{2}^{\prime}, x_{3}, \ldots, x_{N} ; t\right) \psi\left(x_{1}, x_{2}, \ldots x_{N} ; t\right)
\end{array}
$$

and the diagonal part of the two-body density is given by

$\rho^{(2)}\left(x_{1}, x_{2} ; t\right) \equiv \rho^{(2)}\left(x_{1}^{\prime}=x_{1}, x_{2}^{\prime}=x_{2} \mid x_{1}, x_{2} ; t\right)$.

Fig, 4- Fig, 6 plot the results for the spatial two-body density for $g_{d}=0.002,0.02$ and 0.2 respectively. Noninteracting bosons tunnel independently and they are not shown. For inteacting atoms, the effect of two-body correlation is measured by the competition between the non-local interaction and the inter-well coherence. Thus the two-body correlation dynamics expected to be complicated. For larger separation $\left|x-x^{\prime}\right|>>a_{\perp}$ (characteristic length of transverse confinement), the interaction potential varies as $W\left(x-x^{\prime}\right) \sim \frac{1}{\left(x-x^{\prime}\right)^{3}}$ where $\left|x-x^{\prime}\right| \leqslant a_{\perp}$, for small separation the transverse confinement induces a short range cutoff $\alpha_{0}=a_{\perp}$. Snapshots of two-body density $\rho^{(2)}\left(x_{1}, x_{2} ; t\right)$ for $g_{d}=0.002$ are presented in Fig. 4 for different times. At such a weak interaction, the many-body states are fully coherent, with the six bosons clustered in the right well. However, during the tunneling, we observe competition between the correlation due to non-local interaction and the inter-well coherence. With evolution of time, due to the repulsive tail in the interaction, the atoms repel each other and the atoms start to tunnel (snapshot at time $t=10$ ). At larger times (e.g. $t=18$ ), we observe 
four equal bright spots, two diagonal $\left(x=x^{\prime}\right)$ bright spots corresponding to equal number of bosons residing in the two wells, and the other two bright spots along anti-diagonal $\left(x=-x^{\prime}\right)$ expressing the fact that the inter-well coherence is maintained. Thus interatomic correlation and inter-well correlations are maintained in the same scale. During the course of tunneling, we can also verify that at this later time, $\frac{N_{R}(t)}{N} \simeq \frac{N_{L}(t)}{N} \simeq 0.5$ from Fig. 2. At laters time (e.g. $t=29$ ), we observe highly coherent cluster set up in the left well, showing the loss of the inter-well coherence and the prevalence of the interatomic correlations. With time evolving, we observe a rather complex competition between the interatomic correlations in either well and interwell coherence, which results in the Rabi-like oscillations shown in Fig. 2 At longer times, we observe only two bright spots along the diagonal which again corresponds to equal population in two wells $(t=150)$. Comparing with the snapshot at time $t=18$, which also corresponds to equal population in two wells, at time $t=150$ the equal population is obtained at the cost of losing inter-well coherence.

In Fig. 5, we plot the two-body density taking snapshots at different times for $g_{d}=0.02$. In this case, the initially coherent cluster settled in the right well remains same all throughout the time which corresponds to self-trapping as shown in Fig. 2. The interatomic correlation plays the crucial role. However some faded off-diagonal patches appear which signify that exactly $100 \%$ self-trapping is not obtained. It is in good agreement with Fig. 2 which exhibits that for $g_{d}=0.02$, about $90 \%$ self-trapping. Thus the effect of inter-well coherence is very small.

In Fig. 6, we present the two-body density for stronger interaction $g_{d}=0.2$ at different times. Initially at $t=0$ we observe the strong effect of the tails of the repulsive interaction tails: the many-body state is incoherent and diffused unlike the case reported in Fig. 4 and Fig. 5. In Fig. 6 we observe that at time $t=10$ four bright spots are developed which corresponds to the equal population in the two wells. However comparing this with the snapshot at time $t=18$ of Fig. 4 we can clearly identify that the bright spots are now diffused which signifies that three bosons residing in a well feel the effect of the tails of repulsive interaction. Thus atoms are incoherent in each well. With time, the equilibration is almost maintained, however the interatomic coherence in each well depends on time. The equilibration dynamics for $g_{d}=0.2$ is also remarkably different as compared with the case for $g_{d}=0.002$ (Fig. 4). At time $t=150$ of Fig. 4, we observe almost equal population in both wells, but absence of inter-well coherence whereas at the same time in Fig. 6. equilibration is maintained in presence of inter-well coherence. Thus we conclude that the observed dynamics for different choices of interaction strength is fundamentally governed by the interplay between the interatomic correlation and inter-well coherence.

\section{(d) Fragmentation dynamics:}

In mean-field theory, one orbital is macroscopically occupied and the system is condensate. However the phenomenon of fragmentation comes in the picture when more than one orbital is significantly occupied. In our case with $N=6$ particles, we define a quantfier of the observed fragmentation in the dynamics as

$$
F(t)=1-\frac{1}{N} \sum_{i} n_{i}(t)
$$

where $n_{i}(t)$ are the time-dependent natural occupations. Thus $F(t)$ quantifies the contribution coming from other significant orbitals. For $g_{d}=0.002$, and $0.02, F$ is close to zero all throughout the time evolution. However for $g_{d}=0.2$, even at $t=0$, the system is initially fragmented which is also seen in Fig. $7(t=0)$. In a very short time, $F$ increases and reaches a constant value. Then the plateau is maintained for the rest of the time. The plateau corresponds to the equilibration dynamics when the bosons equally distributed in the two wells. In the long-time dynamics (not shown here), we observe the plateau nature is maintained. As $F$ does not increase any more, the occupation in the orbitals remain unchanged: interatomic correlation does not increase further. There is no competition any more between the inter-well coherence and interatomic correlation: thus the equilibration dynamics is maintained for very long time.

\section{Conclusion}

We studied the few-body quantum dynamics of few dipolar bosons in a symmetric double-well. We studied Rabi oscillations in the non-interacting limit and selftrapping and finally equilibration dynamics for strong interaction. The complete dynamics for different choices of the interaction strength is presented through the onebody tunneling dynamics. We further considered the average population imbalance in the two wells as the figure of merit of the tunneling dynamics. The structure in the average population imbalance qualitatively describe the range of interaction strength to have selftrapping, as seen in Fig. 3 . We observed the very interesting dynamics in the two-body density and established the link between the tunneling dynamics and the role played by the interatomic correlation and inter-well 


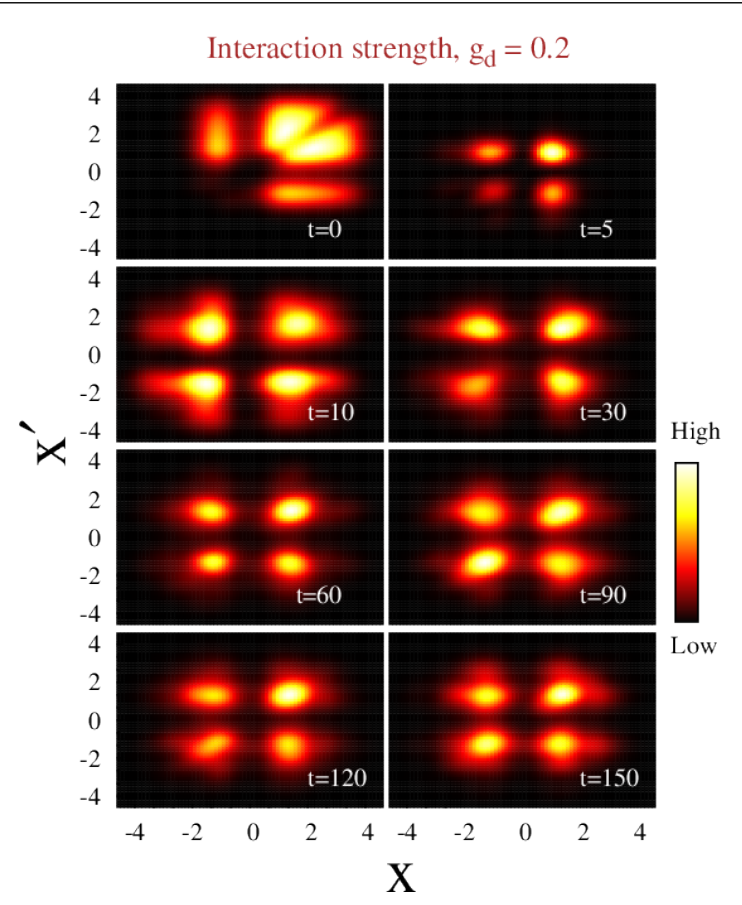

Fig. 6 Time evolution of spatial two-body density for $g_{d}=$ 0.2 .

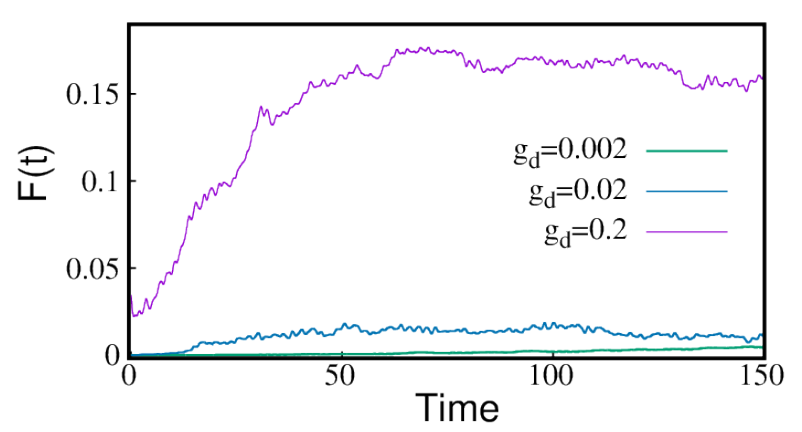

Fig. 7 Plot of $F(t)$ for different interaction strengths. For $g_{d}=0.002$ and 0.02 , system is less fragmented whereas for $g_{d}=0.2$ system is more fragmented and shows a plateau structure.

coherence. The most subtle dynamics is observed at the strong interaction strength when the many-body dynamics exhibits equilibration. Finally we observed that for equilibration dynamics, the many-body state is initially fragmented and then exhibits a plateau structure in the long time dynamics.

Several open questions are suggested by the results presented in this paper. The few-body quantum dynamics gives insights on the phases emerging from the combined presence of the non-local dipolar interaction and of the one-dimensionality. Nevertheless, a full study of the quantum dynamics of dipolar long-range atoms in one-dimensional double-well and the corresponding diagram of dynamical regimes remain to be explores, and one has to see how many-body effects in one-dimension modify the regimes holding for the few-body cases we examined. In partcular, it would be important to address how the non-local nature of the interaction modifies the corresponding results for atoms with shortrange interactions in one-dimensional double-well setups. Moreover, since for very large values of $g_{d}$, dipolar gases exhibits crystallization (see e.g. [102,103]), the double-well dynamics in this regime would be interesting to study. We also mention that in the standard quantum two-mode model one can rewrite the density-density interaction term between the populations of different wells in terms of the local interaction term: therefore, for high barriers, one could ask whether the tunneling dynamics in double-well potentials can be written in terms of an effective contact interaction and how the possibility of performing such a mapping depends on the dimensionality of the systems. We finally mention that the study of the quantum dynamics in presence of both non-local or dipolar and contact interactions in double-well potentials is also to a deserving subject of future investigation.

Acknowledgements R. Roy acknowledges the University Grant Commission (UGC) India for the financial support as a senior research fellow. B. Chakrabarti acknowledges ICTP support where major portion of the work was done.

\section{References}

1. A. Smerzi, S. Fantoni, S. Giovanazzi, and S. R. Shenoy, Phys. Rev. Lett. 79, 4950 (1997).

2. F. S. Cataliotti, S. Burger, C. Fort, P. Maddaloni, F. Minardi, A. Trombettoni, A. Smerzi, and M. Inguscio, Science 293, 483 (2001).

3. M. Albiez, R. Gati, J. Fölling, S. Hunsmann, M. Cristiani, and M. K. Oberthaler, Phys. Rev. Lett. 95, 010402 (2005).

4. T. Schumm, S. Hofferberth, L. M. Andersson, S. Wildermuth, S. Groth, I. Bar-Joseph, J. Schmiedmayer, and P. Krüger, Nature Phys., 1, 57 (2005).

5. T. Anker, M. Albiez, R. Gati, S. Hunsmann, B. Eiermann, A. Trombettoni, and M. K. Oberthaler, Phys. Rev. Lett. 94, 020403 (2005).

6. S. Levy, E. Lahoud, I. Shomroni, and J. Steinhauer, Nature 449, 579 (2007).

7. B. V. Hall, S. Whitlock, R. Anderson, P. Hannaford, and A. I. Sidorov, Phys. Rev. Lett. 98, 030402 (2007).

8. J. Estève, C. Gross, A. Weller, S. Giovanazzi and M. K. Oberthaler, Nature 455, 1216 (2008).

9. L. J. LeBlanc, A. B. Bardon, J. McKeever, M. H. T. Extavour, D. Jervis, J. H. Thywissen, F. Piazza, and A. Smerzi, Phys. Rev. Lett. 106, 025302 (2011).

10. G. Spagnolli, G. Semeghini, L. Masi, G. Ferioli, A. Trenkwalder, S. Coop, M. Landini, L. Pezzé, G. Modugno, M. Inguscio, A. Smerzi, and M. Fattori, Phys. Rev. Lett. 118, 230403 (2017). 
11. K. Xhani, E. Neri, L. Galantucci, F. Scazza, A. Burchianti, K.-L. Lee, C. F. Barenghi, A. Trombettoni, M. Inguscio, M. Zaccanti, G. Roati, and N. P. Proukakis, arXiv: 1905.08893

12. G. Valtolina, A. Burchianti, A. Amico, E. Neri, K. Xhani, J. A. Seman, A. Trombettoni, A. Smerzi, M. Zaccanti, M. Inguscio, and G. Roati, Science 350, 1505 (2015).

13. A. Burchianti, F. Scazza, A. Amico, G. Valtolina, J. A. Seman, C. Fort, M. Zaccanti, M. Inguscio, and G. Roati, Phys. Rev. Lett. 120, 025302 (2018).

14. N. Luick, L. Sobirey, M. Bohlen, V. P. Singh, L. Mathey, T. Lompe, and H. Moritz, arXiv:1908.09776

15. W. J. Kwon, G. Del Pace, R. Panza, M. Inguscio, W. Zwerger, M. Zaccanti, F. Scazza, and G. Roati, arXiv:1908.09696

16. I. Zapata, F. Sols, and A. J. Leggett, Phys. Rev. A 57, R28 (1998).

17. S. Raghavan, A. Smerzi, S. Fantoni, and S. R. Shenoy, Phys. Rev. A 59, 620 (1999).

18. A. Smerzi and A. Trombettoni, Phys. Rev. A 68, 023613 (2003).

19. D. Ananikian and T. Bergeman, Phys. Rev. A 73, 013604 (2006).

20. D. M. Jezek, P. Capuzzi, and H. M. Cataldo, Phys. Rev. A 87, 053625 (2013)

21. J. Javanainen and M. Wilkens, Phys. Rev. Lett. 78, 4675 (1997).

22. S. Raghavan, A. Smerzi, and V. M. Kenkre, Phys. Rev. A 60, R1787(R) (1999).

23. R. Franzosi, V. Penna, and R. Zecchina, Int. J. Mod. Phys. B 14, 943 (2000).

24. A. Smerzi and S. Raghavan, Phys. Rev. A 61, 063601 (2000).

25. R. Gati and M. K. Oberthaler, J. Phys. B 40, R61 (2007).

26. S. Giovanazzi, Estéve, and M. K. Oberthaler, New J. Phys. 10, 045009 (2008).

27. L. Dell'Anna, Phys. Rev. A 85, 053608 (2012).

28. M. Bilardello, A. Trombettoni, and A. Bassi, Phys. Rev. A 95, 032134 (2017).

29. G.-B. Jo, Y. Shin, S. Will, T. A. Pasquini, M. Saba, W. Ketterle, D. E. Pritchard, M. Vengalattore, and M. Prentiss, Phys. Rev. Lett. 98, 030407 (2007).

30. V. Gritsev, A. Polkovnikov, and E. Demler, Phys. Rev. B 75, 174511 (2007).

31. K. Agarwal, E. G. Dalla Torre, J. Schmiedmayer, and E. Demler, Phys. Rev. B 95, 195157 (2017).

32. J. Polo, V. Ahufinger, F. W. J. Hekking, and A. Minguzzi, Phys. Rev. Lett. 121, 090404 (2018).

33. M. Pigneur, T. Berrada, M. Bonneau, T. Schumm, E. Demler, and J. Schmiedmayer, Phys. Rev. Lett. 120, 173601 (2018).

34. J. Polo, R. Dubessy, P. Pedri, H. Perrin, and A. Minguzzi, Phys. Rev. Lett. 123, 195301 (2019).

35. A. I. Streltsov, O. E. Alon, and L. S. Cederbaum, Phys. Rev. A 73, 063626 (2006)

36. A. I. Streltsov, Phys. Rev. A 88, 041602(R) (2013).

37. O. I. Streltsov, O. E. Alon, L. S. Cederbaum, and A. I. Streltsov, Phys. Rev. A 89, 061602(R) (2014).

38. U. R. Fischer, A. U. J. Lode and B. Chatterjee, Phys. Rev.A 91, 063621 (2015).

39. S. Zöllner, H. Meyer, and P. Schmelcher, Phys. Rev. Lett. 100, 040401 (2008).

40. K. Sakmann, A. I. Streltsov, O. E. Alon and L. S. Cederbaum, Phys. Rev. Lett. 103, 220601 (2009).
41. K. Sakmann, A. I. Streltsov, O. E. Alon and L. S. Cederbaum,Phys. Rev. A 82, 013620 (2010).

42. K. Sakmann, A. I. Streltsov, O. E. Alon and L. S. Cederbaum,Phys. Rev. A 89, 023602 (2014).

43. S. K. Haldar and O. E. Alon, New J. Phys. 21, 103037 (2019).

44. A. Griesmaier, J. Werner, S. Hensler, J. Stuhler, and T. Pfau, Phys. Rev. Lett. 94, 160401 (2005).

45. Q. Beaufils, R. Chicireanu, T. Zanon, B. LaburtheTolra, E. Maréchal, L. Vernac, J.-C. Keller, and O. Gorceix, Phys. Rev. A 77, 061601 (2008).

46. M. Lu, N. Q. Burdick, S. H. Youn, and B. L. Lev, Phys. Rev. Lett. 107, 190401 (2011).

47. K. Aikawa, A. Frisch, M. Mark, S. Baier, A. Rietzler, R. Grimm, and F. Ferlaino, Phys. Rev. Lett. 108, 210401 (2012).

48. M. A. Baranov, Phys. Rep. 464, 71 (2008).

49. T. Lahaye, C Menotti, L Santos, M Lewenstein and T Pfau, Rep. Prog. Phys. 72, 126401 (2009).

50. Y. Tang, W. Kao, K.-Y. Li, S. Seo, K. Mallayya, M. Rigol, S. Gopalakrishnan, and B. Lev, Phys. Rev. X 8, 021030 (2018).

51. D. Vodola, L. Lepori, E. Ercolessi, A. V. Gorshkov, and G. Pupillo, Phys. Rev. Lett. 113, 156402 (2014).

52. Z.-X. Gong, M. F. Maghrebi, A. Hu, M. Foss-Feig, P. Richerme, C. Monroe, and A. V. Gorshkov, Phys. Rev. B 93, 205115 (2016).

53. L. Lepori, D. Vodola, G. Pupillo, G. Gori, and A. Trombettoni, Ann. Physics 374, 35 (2016).

54. G. L. Celardo, R. Kaiser, and F. Borgonovi, Phys. Rev. B 94, 144206 (2016).

55. N. Defenu, A. Trombettoni, and S. Ruffo, Phys. Rev. B 94, 224411 (2016); ibid. Phys. Rev. B 96, 104432 (2017)

56. L. Lepori, A. Trombettoni, and D. Vodola, J. Stat. Mech. 033102 (2017).

57. F. Iglói, B. Bla $\beta$, G. Roósz, and H. Rieger, Phys. Rev. B 98, 184415 (2018).

58. B. Bla $\beta$, H. Rieger, G. Roósz, and F. Iglói, Phys. Rev. Lett. 121, 095301 (2018).

59. N. Defenu, T. Enss, M. Kastner, and G. Morigi, Phys. Rev. Lett. 121, 240403 (2018).

60. L. Lerose, B. Zunkovic, A. Silva, and A. Gambassi, Phys. Rev. B 99, 121112 (2019).

61. L. Santos, G. V. Shlyapnikov, P. Zoller and M. Lewenstein, Phys. Rev. Lett. 85, 1791 (2000).

62. S. Sinha and L. Santos, Phys. Rev. Lett. 99, 140406 (2007).

63. R. Citro, E. Orignac, S. De Palo, and M.-L. Chiofalo, Phys. Rev. A 75, 051602 (2007).

64. P. Bader and U. R. Fischer, Phys. Rev. Lett. 103, 060402 (2009).

65. N. Henkel, F. Cinti, P. Jain, G. Pupillo and T. Pohl, Phys. Rev. Lett. 108, 265301 (2012).

66. F. Cinti, T. Macrì, W. Lechner, G. Pupillo, and T. Pohl, Nature Communications 5, 3235 (2014).

67. S. De Palo, R. Citro, and E. Orignac, Phys. Rev. B 101, $045102(2020)$.

68. M. Abad, M. Guilleumas, R. Mayol, M. Pi, and D. M. Jezek, Phys. Rev. A 84, 035601 (2011).

69. M. Abad, M. Guilleumas, R. Mayol, M. Pi and D. M. Jezek, Euro. Phys. Lett., 9410004 (2011).

70. A. Gallem, M. Guilleumas, R. Mayol, and A. Sanpera, Phys. Rev. A 88, 063645 (2013).

71. M. Asad-uz-Zaman and D. Blume, Phys. Rev. A 80, 053622 (2009).

72. G. Mazzarella and L. Dell'Anna, EPJ ST 217, 197 (2013). 
73. S. K. Haldar and O. E. Alon, Chem. Phys. 509, 72 (2018).

74. S. K. Haldar and O. E. Alon, J. Phys.: Conf. Series 1206, 012010 (2019).

75. M. Olshanii, Phys. Rev. Lett. 81, 938 (1998).

76. A. J. Leggett, Rev. Mod. Phys. 73, 307 (2001).

77. A. I. Streltsov, O. E. Alon, and L. S. Cederbaum, Phys. Rev. Lett. 99, 030402 (2007).

78. O. E. Alon, A. I. Streltsov, and L. S. Cederbaum, Phys. Rev. A 77, 033613 (2008).

79. E. Fasshauer and A. U. J. Lode, Phys. Rev. A 93, 033635 (2016).

80. Rui Lin et. al. Quantum Sci. Technol. 5024004 (2020).

81. A. U. J. Lode, M. C. Tsatsos, E. Fasshauer, R. Lin, L. Papariello, P. Molignini, C. Lévêque, and S. E. Weiner, MCTDH-X:The time-dependent multiconfigurational Hartree for indistinguishable particles software, http://ultracold.org (2018).

82. A. U. J. Lode, Phys. Rev. A 93, 063601 (2016).

83. B. Chatterjee, C. Lévêque, J. Schmiedmayer, and A. U. J. Lode Phys. Rev. Lett. 125, 093602 (2020).

84. Budhaditya Chatterjee and Axel U. J. Lode Phys. Rev. A 98, 053624 (2018).

85. Budhaditya Chatterjee et. al., New J. Phys. 21, 033030 (2019).

86. J. Grond, J. Schmiedmayer, and U. Hohenester, Phys. Rev. A 79, 021603(R) (2009).

87. J. Grond, T. Betz, U. Hohenester, N. J. Mauser, J. Schmiedmayer, and T. Schumm, New J. Phys. 13, 065026 (2011)

88. L. Cao, S. Krönke, O. Vendrell, and P. Schmelcher, J. Chem. Phys. 139, 134103 (2013).

89. R. Schmitz, S. Krönke, L. Cao, and P. Schmelcher, Phys. Rev. A 88, 043601 (2013).
90. J. M. Schurer, A. Negretti, and P. Schmelcher, New J. Phys. 17, 083024 (2015).

91. A. U. J. Lode, B. Chakrabarti, and V. K. B. Kota, Phys. Rev. A 92, 033622 (2015).

92. A. U. J. Lode and C. Bruder, Phys. Rev. Lett. 118 013603 (2017).

93. C. Lévêque and L. B. Madsen, New J. Phys. 19, 043007 (2017).

94. G. C. Katsimiga, S. I. Mistakidis, G. M. Koutentakis, P. G. Kevrekidis, and P. Schmelcher, New J. Phys. 19, 123012 (2017).

95. R. Roy, A. Gammal, M. C. Tsatsos, B. Chatterjee, B. Chakrabarti, and A. U. J. Lode, Phys. Rev. A 97, 043625 (2018).

96. R. Roy, C. Lévêque, A. U. J. Lode, A. Gammal, and B. Chakrabarti, Quantum Reports 1, 304 (2019).

97. S. Bera, R. Roy, A. Gammal, B. Chakrabarti, and B. Chatterjee, J. Phys. B 52, 21 (2019).

98. J.H.V. Nguyen, M.C. Tsatsos, D. Luo, A.U.J. Lode, G.D. Telles, V.S. Bagnato, and R.G. Hulet Phys. Rev. X 9, 011052 (2019).

99. A. Lode, C. Lévêque, L. Madsen, A. Streltsov, and O. E. Alon, rev. Mod. Phys., 92011001 (2020).

100. P. Kramer and M. Saracento, Geometry of the timedependent variational principle (Springer, Berlin, 1981).

101. H.-J. Kull and D. Pfirsch, Phys. Rev. E 61, 5940 (2000).

102. T. Macrì, F. Maucher, F. Cinti, and T. Pohl, Phys. Rev. A 87, 061602(R) (2013).

103. S. Bera, B. Chakrabarti, A. Gammal, M. C. Tsatsos, M. L. Lekala, B. Chatterjee, C. Lévêque, and A. U. J. Lode, Sci. Rep. 9, 17873 (2019). 